\title{
Observations of Local ISM Emission with the Berkeley EUV/FUV Shuttle Telescope
}

\author{
Christopher Martin and Stuart Bowyer \\ Space Science Laboratory, Iniversity of California, Berkeley
}

ABSTRACT

I'he Berkeloy Extreme Litraviolet, F'ar Ultraviolet Snuttle Telescope (BEST) will be launched on the Space Shuttle in November, 1984, as part of the NASA LVX project. The Berkeley spectrometer will make observations of the cosmic diffuse background in the 600-1900 $\AA$ band, with a spectral resolution of $10 \AA$. The sensitivity and spectral resolution of the instrument make it ideal for the study of components of the interstellar medium in the $10^{4}$. $10^{6} \mathrm{~K}$ range.

\section{Introduction}

A number of observations have been made of the diffuse ultraviolet background, but have been subject to the adverse effects of stellar contamination, airglow and geocoronal emission, and high internal background, as well as generally marginal sensitivity. Combined with significant differences in observation platforms and instrument design, these problems have caused serious discrepancies to appear between different observers (cf. review by Paresce and Jaksbsen, 1990), even when observing identical parts of the sky (Paresce, Mckee, and Bowyer, 1980). A primary objective of the first mission is to perform coobservations with the Johns Hopkins Lltraviolet Spectrometer, and attempt to resolve these differences. Beyond this goal, detailed and sensitive measurements of the diffuse background, free of systematic errors, should hold considerable information about a variety of processes originating in the interstellar mediurn.

\section{Instrument Design}

The Berkeley spectrometer has been designed primarily as a nebular spectrophotometer, which is optimized by a high geometric area-field of view product, and encorporates a fast $(f / 2.0)$ off-axis paraboloid, Rowland spectrograph combination. Figure 1 shows a schematic view of the instrument, and Table 1 summarizes the instrument characteristics. The use of photon counting microchannel plate detectors with a wedge-and-strip readout (Martin et al. 1981) and a high yield Csl photocathode (Martin and Bowyer, 1982) maximizes the throughput and minimizes the detector dark background. The twodimensional imaging of the detectors allows complete spectral multiplexing over the 600-1900 \& range.

Stellar contamination is avoided by imaging along the long axis of the spectrometer entrance slit, which is accomplished with a toroidal grating and the 2-D detcctors. In addition, à superpolished mirror and holographically ruled grating eliminate scattering into the entrance slit or within the spectrometer. A star camera is used to reconstruct the true aspect post-mission to determine the positions of all stars, and the CsI photocathode efficiency drops rapidly longward of $1900 \AA$ eliminating nore ubiquitous $F$ stäs from scattering àn effectirg the spectrum. 
Scattering from geocoronal and airglow lines that would otherwise be imaged into $10 \AA$ of the spectrum has been minimized by using a holographic grating. Filters are placed at the detectors to eliminale the residual scattered Lyman $\alpha$ 1216, OI 1304 and $1356 \AA$ emission. A test filter is periodically interposed at the entrance slit which permits a measurement of the residual Lyman $\alpha$ in the $\operatorname{EUV}(600-1150 \AA)$ band.

The effect of detector internal background is minimized by using a fast optical system, and the level is measured regularly on orbit using a rotating shutter mechanism. Background inducing particle ingress is minimized by using a gas tight spectrometer box and ion-repeller grid at the entrance slit. All operations will be performed outside of areas of high energetic particle flux.

Shuttle glow phenomena could interfere with the measurements, but the spectral resolution, time resolution, photon counting nature of the detectors, and the use of filters against ol lines should reduce the impact of such emission.

The instrument sensitivity to continuum and line emission are shown in Figure 2, along with the sensitivity of a number of other instruments, including IUE, and the Voyager UVS. The FUV continuum sensitivity (expressed as a minimum detectable flux for one orbital night integration) is a factor of 30-50 lower than the lowest level detected $\left(\sim 300 \mathrm{ph} \mathrm{cml}^{-2} \mathrm{~s}^{-1} \mathrm{~A}^{-1} \mathrm{sr}^{-1}\right)$ (Paresce, McKee, and Bowyer, 1980; Jakobsen et al., 1984). The EUV continuum sensitivity is $\sim 300-1000 \mathrm{ph} \mathrm{cm}^{-2} \mathrm{~s}^{-1} \mathrm{~A}^{-1} \mathrm{sr}^{-1}$, for an integration of $10^{4}$ seconds, which will be achicved by summing the data from ten single orbit observations. The FUV line sensitivity is limited by the level of the FUV continuum, and is $\sim 500 \mathrm{ph} \mathrm{cm}^{-2} \mathrm{~s}^{-1}$ $\mathrm{sr}^{-1}(0.004 \mathrm{Ry})$ for a $1000 \mathrm{sec}$ integration.

\section{Observations of ISM Emission}

I'he high sensitivity and moderate spectral resolution of the Berkeley spectrometer should permit the separation of a variety of interstellar emission processes which have unique spectral signatures. In general, observations of ISM emission will give information about spatial exterision, morphology, and emission measures which lead to filling factors when compared with measurements that make a linear average of line of sight densities.

Dust is detectable via scattered starlight (Paresce and Jakobsen, 1980), and the spectrum will include effects of wavelength dependent albedo and dust size spectra. Targets in directions of anomalous gas-to-dust ratios may reveal clues about the origin of these anomalies, including varying dust properties and the presence of molecular hydrogen.

Molecular hydrogen should produce FUV fluorescence as a natural byproduct of its formation/destruction cycle (Jakobsen, 1982). Observable fluorescence should be produced in clouds of density greater than $\mathrm{n}_{H} \sim 10\left(10^{20} / \mathrm{N}_{H}\right)$, so that lypical diffuse clouds should exhibil delectable ennission. Since this process is density sensitive, the levels detecled are indicative of the density, morphology and filling factor of diffuse clouds.

Warm ionized gas at $10^{4} \mathrm{~K}$ will produce 2-photon continuum emission, which can be directly correlated with observations of recombinant $\mathrm{Ha}$ emission (Deharveng et al, 1982). (Gas at "semi-torrid" temperatures $\sim 10^{4.8-5.8}$ should exhibit collisionally excited line emission from a variety of metal resonance transitions (Jakobsen and Paresce, 1981). Feldman et al. (1981) subsequently made tentative identification of this type of line emission from Si IV $1398 \AA$, N IV $1487 \AA, C, I V 1549 \AA$, and $O$ III $1663 \AA$, in the direction of the north galactic pole. Paresce et al. (1983) have interpreted this emission as arising from the same 
$10^{5.2} \mathrm{~K}$ gas that could accuunt for observed EUV emission. The derived constraints on the EM vs. temperature are shown in Figure 3, along with the EM required to produce detectable lines for the Berkeley spectrometer. A variety of lines should be detectable in more than one ionization state, allowing the determination of temperature (or temperature distribution), filling factors (when compared to absorption line measurements), spatial distribution, and possibly the effects of non-equilibrium ionization.

\section{References}

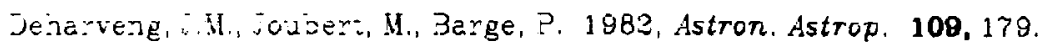

Eedran. J.J., 9rune, W.H and Henry, R.C. 1981, Ap. J. Lett, 248, L51.

iakosen, ?., and Paresce, E. 1981, Astron. Astrop. 86, 23.

IExozsen, ?. 1882, Astron. Astrop. B0,67.

iakobsen. P., Bowyer, S., Kimble, R., Jelinsky, P., Grewing, M., Kramer, G 1984, Astron. Astrop., Subr.t.ted

Martin, C., Jelinsky, P., Lampton, M., Malina, R.F., and Anger, H.O, 1981, Rev. Sci. Instrum. 52, 1067.

Martin, C, and Bowyer, S. 1882, Applied Optics 21, 4206.

Paresce, F, and Jakobsen, P. 1980, Nature 288, 119.

Paresce, F., McKee, C., and Bowyer, S. 1880, Ap. J. 240, 387.

Paresce, F. and Stern, R, 1981,Ap.J. 247, 89.

Paresce. F., Monsignori Fossi, B.C., Landini, M. 1883., Ap. J. Lett. 266, L107.

Table I

BEST Instrument Summary

Objective
Grating
Grating radius
Groove density
Dispersion
Mirror/Grating Coating
Field of View
Bandpass
Resolution
Detectors
Detector readout
Photocathode
Filters

Sensitivity
Continuum
Line

$15 \mathrm{~cm} \mathrm{f} / 2$ off-axis paraboloid Toroidal holographic $30 \mathrm{~cm} \times 28.9 \mathrm{~cm}$ $2000 \mathrm{l} / \mathrm{mm}$ $16.7 \AA / \mathrm{mm}$ $\mathrm{Al}+150 \AA \mathrm{MgF}$

$0.1 \times 4.0$ degrees

$600-1140 \AA$ $1350-1930 \AA$ $10 \AA$

2 microchannel plate wedge \& strip anude $6000 \AA \mathrm{Cs}$ Tin $(1500 \AA) 600-750 \AA$ Indium (1600 $\AA$ ) 750-1150 $\operatorname{BaF}(2 \mathrm{~mm}) 1350-1930 \AA$

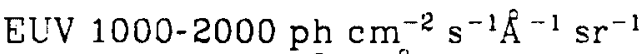
FUV 4-10 ph $\mathrm{cr}^{-2} \mathrm{~s}^{-1} \AA^{-1} \mathrm{sr}^{-1}$

EUV $10^{4} \mathrm{ph} \mathrm{cm}^{-2} \mathrm{~s}^{-1} \mathrm{sr}^{-1}$

FUV 400-600 ph $\mathrm{cm}^{-2} \mathrm{~s}^{-1} \mathrm{sr}^{-1}$ 

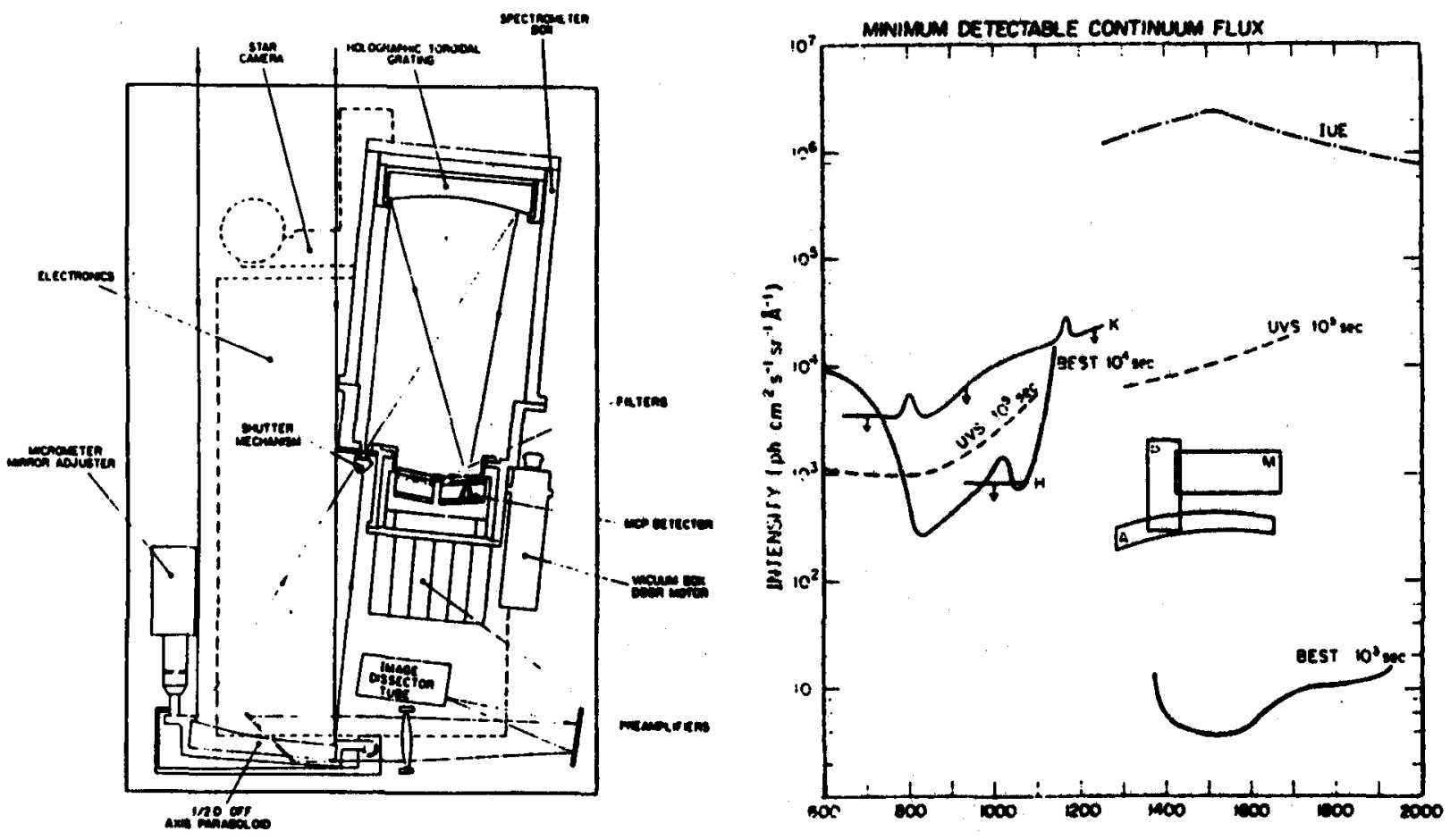

Figure 1.

Figure 2. Sensitivity (minimum detectable flux) for $10^{3} \mathrm{sec}$ (EUV) are $10^{4} \mathrm{sec}$ (EUV),

Schematic of Berkeley Spectrometer with Voyager UVS and IUE sensitivities.

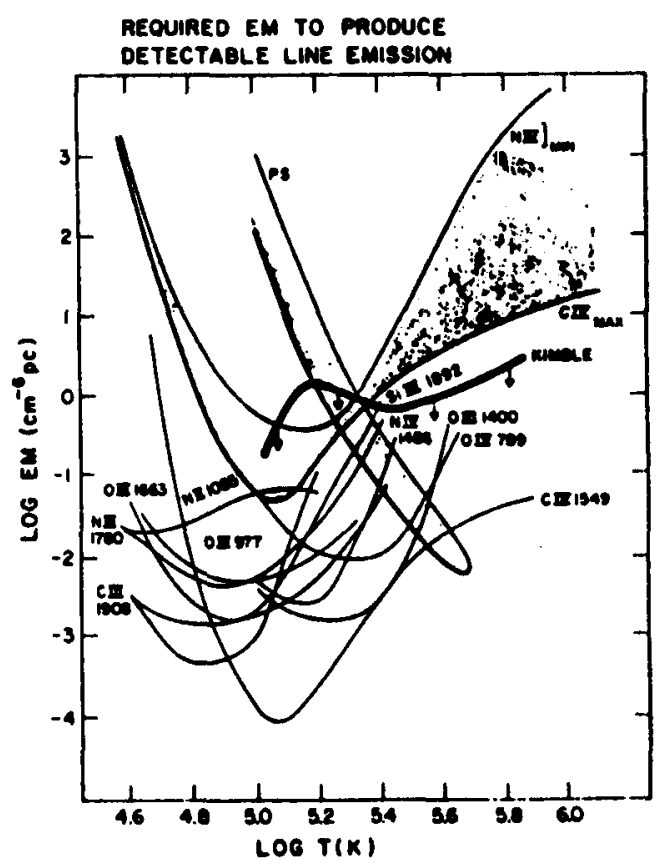

Figure 3. Plasma constraints derived by Paresce, et al 1983 from EUV line detections by Feldman et al 1983 and Paresce and Stern, 1981, and Kimble, 1983 (U.C. Berkeley PhD), along with detectable line emission. 